\title{
Study on the Motivational and Physical Effects of Two VR Exergames
}

\begin{abstract}
Head-mounted displays for accessing virtual reality have in the past years begun to achieve their commercial potential. Gameplay using the provided game controls and space, often results in increased mobility due to this the games may have unintentional health benefits. In this study we compare two distinct exergames. We conducted a study with 17 participants and collected mixed data in the form of heart rate, interviews and accelerometer data, to assess the exercise benefit of these games. Virtual reality games can be played for exercise purposes. Participants were more eager to recommend others to exercise with exergames than exercise themselves using them. With a more hectic game, it was shown that their heart rate increased, and they moved a lot during the game sessions.
\end{abstract}

Keywords - exergames, virtual reality, exercise benefits, mixed methods

\section{INTRODUCTION}

The aim of this study is to detect exercise motivation by having users' play virtual reality (VR) exergames while measuring physical intensity. Users were interviewed and had their heart rate and movements measured. The goal is to see if VR games have physical effects on a user and if they enjoy exercising with VR games. In this study we aim to answer the following research questions; Do VR-based exergames have physical benefits and do users like to exercise by playing a VR-based exergame?

\section{BACKGROUND}

\section{A. Exergames}

Exergames are games combining exercise and gameplay. The term exergame is widely used when video games are played to promote physical activity [1]. Fitzgerald et al. [2] have shown that exergames can be used successfully also in therapy. Their results show that patients are more motivated for training when comparing to those who do similar exercises at a class with a therapist [2].

\section{B. Exercise intensity}

Heart rate measurements have long been used to monitor exercise intensity [3]. Although the maximum heart rate varies greatly between individuals, it can be estimated without measurement if the person's age is known. Robergs et al. [4] suggest the formula:

$$
H R(\text { maximum })=205.8-(0.685 \times \text { age })
$$

The United States Center of Disease Control [5] recommends target heart rates of $50-70 \%$ for moderate exercise and 70 - $85 \%$ for vigorous exercise. Another method used to gauge the exercise intensity is the heart rate reception [6] with formula:

$$
H R(\text { reserve })=H R(\text { maximum })-H R(\text { rest })
$$

Relative heart rate can then be used to detect the changes in the heart rates with formula:

$$
H R(\text { relative })=\frac{H R(\text { atm })-H R(\text { rest })}{H R(\text { reserve })} \times 100 \%
$$

\section{EXPERIMENTAL SETUP}

The test took place in controlled environment in the University of Oulu. HTC Vive HMD was used. 17 participants played two different games and their heart rate and movement were measured while they were playing. Movement data was collected using a capture program which was running parallel to the VR games. The program sampled the position and orientation data from all the three devices at a rate of $5 \mathrm{~Hz}$. Fitbit Charge HR bracelet was used to collect heart rate data. Participants were interviewed before game sessions, between and after game session.

We used two different commercial games, QuiVR and BeatSaber. Participants played games in different order. QuiVR is an arcade-style archery simulation. In BeatSaber, a player must cut moving colored blocks and dodge around obstacles.

\section{RESULTS}

From 17 test users, heart rate data was collected from 14 participants. Average heart rate data is shown in Fig. 1. From Fig. 1 can be seen the average of the users' heart rate changes during game sessions. Relative heart rates increased faster when participants were playing BeatSaber than QuiVR.

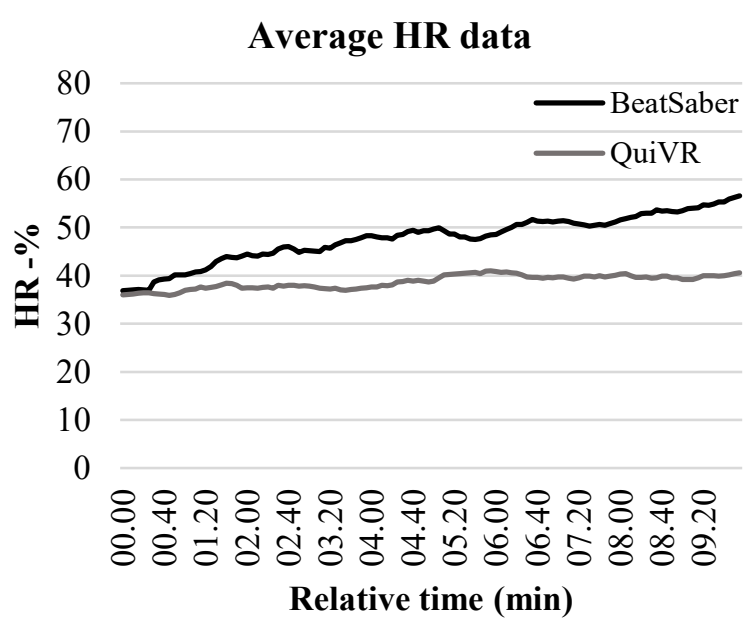

Fig. 1. Average heart rates of participants during each game session. Gray line is for BeatSaber game and the black line is for QuiVR game.

Users felt that BeatSaber was more intense game than QuiVR (Fig. 2). 


\section{Self evaluation of game session intensity}

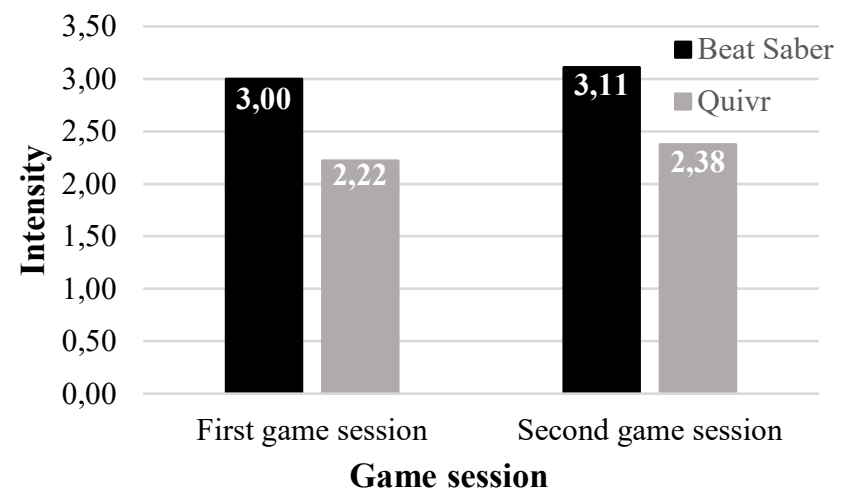

Fig. 2. Users' self evaluation of the intensity of the game session. Half of the users played BeatSaber first and QuiVR next, half vice versa.

Users' movements were collected during game sessions (Fig 3). Movement data is the sum of the movements of HMD and motion controllers. Data was relative and it was compared against the data from the user who moved most.

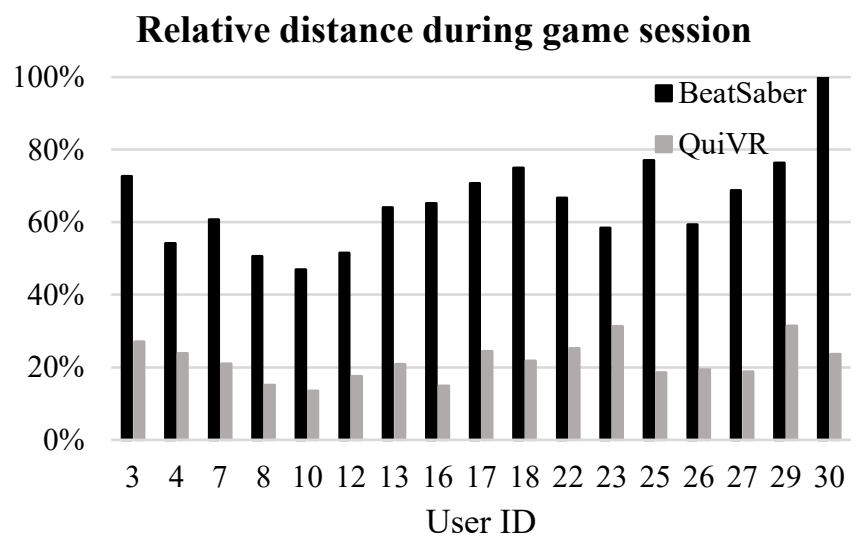

Fig. 3. Participant's relative distance moved during each game sessions. Gray bar is for BeatSaber game and the black bar is for QuiVR game.

\section{DISCUSSION AND CONCLUSIONS}

In this study, we compared two different VR games (BeatSaber and QuiVR) to explore their motivational effects to exercise and their physical benefits. Our results show that both games have potential in motivating exercise. Even if the subjects felt unsure if VR games could motivate them to exercise more, they could see themselves recommending VR games as exercise for someone else. However, most participants viewed the experience positively and said they had fun while playing.

Results from the interviews showed that participants would play VR games just for fun with an average of 4.6 out of five. When participants were asked if they would play VR games for exercise purpose, they rated it as an average of 2.9 out of five. When they were asked if playing VR games would encourage them to exercise more, they rated it as an average of 2.6 out of five. Participants enjoyed playing VR games and they said after game sessions that playing was fun and some said it was also challenging. Even participants gave neutral answers (average 2.6 out of five) to question "if VR games would encourage them to exercise more", they still would recommend VR playing as an exercise to others with an average 3.4 out of five.

As it can be seen in Fig. 1., participants' heart rate in BeatSaber game raised during the whole game session and it could have been raised even higher if the game session had been continued for more than 10 minutes. In QuiVR game session heart rates of the participants did not raise as clearly, and it is hard to predict if they would have been raised if the game session was longer. The reason why is that, participants moved multiple times more (Fig. 3.) in BeatSaber game session than in QuiVR game session. The difference in average heartrate was small (Fig 1.), but the game that required more movement (Fig. 3) increased the heartrate as the game progressed. This proves, perhaps obviously so, that BeatSaber is better for aerobic exercise and QuiVR, which was considered less intense would be better suited for training for muscle tone. This study also showed that the combination of lightweight HR tracker, in-built sensors in HMD, and selfassessment, are enough in order to observe differences between two distinct exergames and the type of exercise they actuate. However, in the future, we will consider complementing our biometric measurements with electromyography. It would also be interesting to see how a VR-based exercise regimen might affect exercise motivation in long term. We would also like to explore if there is relation between exercise motivation with VR games and background with playing videogames.

\section{ACKNOWLEDGMENT}

[Anonymized]

\section{REFERENCES}

[1] L. Rebenitsch and C. Owen, "Review on cybersickness in applications and visual displays," Virtual Reality, vol. 20, no. 2, pp. 101-125, 2016.

[2] D. Fitzgerald, N. Trakarnratanakul, B. Smyth, and B. Caulfield, "Effects of a wobble board-based therapeutic exergaming system for balance training on dynamic postural stability and intrinsic motivation levels," journal of orthopaedic \& sports physical therapy, vol. 40, no. 1, pp. 11-19, 2010.

[3] J. Achten and A. E. Jeukendrup, "Heart rate monitoring," Sports medicine, vol. 33, no. 7, pp. 517-538, 2003.

[4] R. A. Robergs and R. Landwehr, "The surprising history of the" HRmax $=220$-age" equation," Journal of Exercise Physiology Online, vol. 5, no. 2, pp. 1-10, 2002.

[5] M Gulati, LJ Shaw, MS Lauer, RA Thisted, HR Black, CNB Merz, R Wicklund, AJ Al-Hani, and MF Arnsdorf. 2005. Heart rate response to exercise stress testing in asymptomatic women: The St. James women take heart project. U885-U885.

[6] M.J. Karvonen. The effects of training on heart rate: a longitudinal study. Ann Med Exp Biol Fenn, 35:307-315, 1957. 